Scheduled for ApJ, v536n1, Jun 10, 2000

\title{
Dithering Strategies for Efficient Self-Calibration of Imaging Arrays
}

\author{
Richard G. Arendt ${ }^{1}$, D. J. Fixsen ${ }^{1}$, \& S. Harvey Moseley \\ Laboratory for Astronomy and Solar Physics, \\ Code 685, NASA GSFC, Greenbelt, MD 20771; \\ arendt, fixsen, moseley@stars.gsfc.nasa.gov
}

\begin{abstract}
With high sensitivity imaging arrays, accurate calibration is essential to achieve the limits of detection of space observatories. One can simultaneously extract information about the scene being observed and the calibration properties of the detector and imaging system from redundant dithered images of a scene. There are large differences in the effectiveness of dithering strategies for allowing the separation of detector properties from sky brightness measurements. In this paper, we quantify these differences by developing a figure of merit (FOM) for dithering procedures based on their usefulness for allowing calibration on all spatial scales. The figure of merit measures how well the gain characteristics of the detector are encoded in the measurements, and is independent of the techniques used to analyze the data. Patterns similar to the antenna arrangements of radio interferometers with good $u-v$ plane coverage, are found to have good figures of merit. We present patterns for both deep surveys of limited sky areas and for shallow surveys. By choosing a strategy that encodes the calibration in the observations in an easily extractable way, we enhance our ability to calibrate our detector systems and to reach the ultimate limits of sensitivity which are required to achieve the promise of many missions.
\end{abstract}

Subject headings: instrumentation: detectors — methods: data analysis — techniques: photometric

\section{Introduction}

In order to achieve their required performance, many observing systems must observe with sensitivities near their confusion limits. Many instruments are capable of reaching these limits in crowded stellar fields such as the Galactic center. Future instruments such as the Infrared Array Camera (IRAC) and the Multiband Imaging Photometer (MIPS) on the Space Infrared Telescope Facility (SIRTF) and those planned for the Next Generation Space Telescope (NGST) will be able to reach limits in which the confusion of extragalactic sources becomes significant. In general, the measurement noise is determined by both the statistical fluctuations of the photon flux and uncertainties in detector gain and offset. Any successful calibration procedure must determine these detector parameters sufficiently accurately so that their uncertainties make small contributions to the measurements errors compared to those of the

\footnotetext{
${ }^{1}$ Raytheon ITSS
} 
background fluctuations. If the science done with the instrument requires substantial spatial or temporal modeling, calibration requirements become more demanding, ultimately requiring similar integration time for observation and calibration as in the case of the COBE FIRAS instrument (Mather et al. 1994; Fixsen et al. 1994). Additionally, in such cases robust error estimators are often needed. A common method to determine the instrument calibration is to look at known calibration scenes (e.g. a dark shutter, an illuminated screen, or a blank region of sky) of different brightnesses to deduce gain and offset of each detector pixel. This requires a well characterized calibration source and often a change in instrument mode to carry out the measurement. This procedure may introduce systematic errors relating to the extrapolations from the time and conditions of the calibration observations to the time and conditions of the sky observations and from the intensity (and assumed flatness) of the calibration source to the intensity of the observed sky. A different approach is to use the measurements of the sky alone to extract the calibration data for the system. By using the sky observations for calibration, the systematic errors introduced by applying a calibration derived from a distinctly different data set are eliminated. Such methods require a set of dithered images, where a single sky location is imaged on many different detector pixels.

Typical CCD and IR array data reduction procedures for a set of dithered images make use of a known or measured dark frame $\left(F^{p}\right)$ and derive the flat field $\left(G^{p}\right)$ through taking the weighted average or median value of all data $\left(D^{i}\right)$ observed by each detector pixel $p(i \in p)$ in a stack of dithered images (e.g. Tyson 1986, Tyson \& Seitzer 1988, Joyce 1992, Gardner 1995). The least squares solution of

$$
\mathcal{D}^{i}=G^{p} S^{0}
$$

where $\mathcal{D}^{i}=D^{i}-F^{p}(i \in p)$ and $S^{0}$ is the perfectly flat sky intensity, for $G^{p}$, the flat field, is

$$
G^{p}=\frac{\sum_{i \in p} \mathcal{D}^{i} W_{i}}{\sum_{i \in p} W_{i}} \frac{1}{S^{0}}
$$

which is simply the weighted average of the data collected by each detector pixel normalized by the constant sky intensity (to be determined later though the absolute calibration of the data). The weights, $W_{i}$, are normally determined by the inverse variance of the data, but may also be set to zero to exclude sources above the background level. The use of the median, instead of the weighted average, also rejects the outliers arising from the observations of real sources instead of the flat background, $S^{0}$, and formally corresponds to a minimization of the mean absolute deviation rather than a least squares procedure. In either form, this method requires observations of relatively empty fields where variations in the background sky level are not larger than the faintest signal that is sought. Thus, throughout this paper we refer to such procedures as "flat sky" techniques. As instrumentation improves and telescope sensitivity increases, this condition is becoming harder to fulfill. In fields at low Galactic latitude, stellar and nebular confusion can be unavoidable, and at high latitude deep imaging (particularly in the infrared) is expected to reach the extragalactic confusion limit. In such cases, because of the complex background, and in other cases where external influences (e.g. moonlight, zodiacal light) create a sky background with a gradient, the flat sky approach does not work and a more comprehensive approach must be used.

Such an approach has been presented by Fixsen et al. (2000) who describe the general least squares solution for deriving the sky intensity $S^{\alpha}$ at each pixel $\alpha$, in addition to the detector gain (or flat field) $G^{p}$ and offset (or dark current + bias) $F^{p}$ at each detector pixel $p$, where each measurement, $D^{i}$, is represented by

$$
D^{i}=G^{p} S^{\alpha}+F^{p}
$$

(Throughout this paper we refer to the procedure described by Fixsen et al. (2000) as the "least squares" procedure.) They show how the problem of inverting large matrices can be circumvented, and how the 
formulation of the problem allows for explicit tracking of the uncertainties and correlations in the derived $G^{p}, F^{p}$, and $S^{\alpha}$. Fixsen et al. also show that although the formal size of the matrices used in the least squares solution increases as $P^{2}$, where $P$ is the number of pixels in the detector array, the number of non-zero elements in these matrices increases only as $M \times P$, where $M$ is the number of images in the data set. In practice, the portion of the least squares solution for the detector gains and offsets is calculated first, and then the data are corrected to produce images of the sky $\left(S^{\alpha}\right)$ that are registered and mapped into a final single image. Because this approach explicitly assumes a different sky intensity at each pixel, the crowded or confused fields that can cause the flat sky technique to fail are an aid to finding the least squares solution. Thus, the need for chopping away from a complex source in order to observe a blank sky region is eliminated. The simultaneous solution for both the detector gain and offset also eliminates the need for dark frame measurements, although if dark frame measurements are available then they can be used with the other data to reduce the uncertainty of the procedure. We note that this general least squares approach may also be applied in non-astronomical situations (e.g. terrestrial observing) where complex images are the norm.

The flat sky technique works well in situations where all detector pixels spend most of the time observing the same celestial calibration source, namely the flat sky background. For this technique, dithering is required only to ensure that all pixels usually do see the background. Because all pixels have observed the same source, the relative calibrations of any two pixels in the detector are tightly constrained, regardless of the separation between the pixels, i.e.

$$
\frac{G^{1}}{G^{2}}=\frac{G^{1} S^{0}}{G^{2} S^{0}}=\frac{\mathcal{D}^{1}}{\mathcal{D}^{2}}
$$

However, in the more general least squares solution of Fixsen et al. (2000), each sky pixel $\left(S^{\alpha}\right)$ represents a different celestial calibration source. The only pixels for which the relative calibrations are tightly constrained are those that through dithering have observed common sky pixels. Pixels that do not observe a common sky pixel are still constrained, though less directly, by intermediate detector pixels that do observe common sky pixels. For example, the relative calibration of detector pixels 1 and 3 which observe sky pixels $\alpha$ and $\beta$ respectively, but no common sky pixels, may be established if an intermediate detector pixel 2 does observe both sky pixels $\alpha$ and $\beta$, i.e.

$$
\frac{G^{1}}{G^{3}}=\frac{G^{1} S^{\alpha}}{G^{2} S^{\alpha}} \frac{G^{2} S^{\beta}}{G^{3} S^{\beta}}=\frac{\mathcal{D}^{1 \alpha}}{\mathcal{D}^{2 \alpha}} \frac{\mathcal{D}^{2 \beta}}{\mathcal{D}^{3 \beta}} .
$$

Other detector pixels might require multiple intermediate pixels to establish a relative calibrations. As the chain of intermediate pixels grows longer, the uncertainty of the relative calibration of the two pixels also grows. Therefore, when applying the least squares solution, the exact dither pattern becomes much more important than in the flat sky technique. For the least squares solution to produce the smallest uncertainty, the dither pattern should be one that establishes the tightest correlations between all pairs of detector pixels using a small number of dithered images. Even if one is only interested in small scale structure on the sky (e.g. point sources), it is still important to have the detector properly calibrated on all spatial scales to prevent large scale detector variations from biasing results derived for both sources and backgrounds imaged in different parts of the array.

Whether obtained by flat sky, least squares, or other techniques, the quality of the calibration is ultimately determined by its uncertainties. For the least squares solution of Fixsen et al. (2000), understanding the uncertainties is relatively straight forward, because it is a linear process, i.e. $P^{\alpha}=L_{i}^{\alpha} D^{i}$ where $P^{\alpha}$ is the set of fitted parameters, $D^{i}$ is the data, and $L_{i}^{\alpha}$ is a linear operator. Then, given a covariance 
matrix of the data, $\Sigma^{i j}$, the solution covariance matrix is $V^{\alpha \beta}=L_{i}^{\alpha} L_{j}^{\beta} \Sigma^{i j}$. For a nonlinear process such as a median filter the uncertainties are harder to calculate. The diagonal terms of the covariance matrix of the solution might be sufficiently well approximated by Monte Carlo methods, but the off-diagonal components are far more numerous and often more pernicious as the effects can be more subtle than the simple uncertainty implied by the diagonal components. For this reason the off-diagonal components are often ignored. Creating final images at subpixel resolution (e.g. "drizzle", Fruchter \& Hook 1998) may introduce additional correlations beyond those described by the covariance matrix, and disproportionately increase the effects of the off-diagonal elements of the correlation matrix. Accurate knowledge of all these uncertainties is especially important for studies that seek spatial correlations within large samples, such as deep galaxy surveys or studies of cosmic backgrounds, so that any detected correlations are certifiably real and not artifacts caused by the calibration errors and unrecognized because of incomplete or faulty knowledge of the uncertainties.

Table 1 itemizes some of the features of each data analysis technique. The remainder of this paper is concerned with characterizing what makes a dither pattern good for self-calibration purposes using the least squares solution. We present a "figure of merit" (FOM) which can be used as a quantitative means of ranking the suitability of different dither patterns $(\S 2)$. We then present several examples of good, fair and poor dither patterns $(\S 3)$, and investigate how changes to the patterns affect their FOM. In $\S 4$, we show how dithered data can be collected in the context of both deep and shallow surveys. We also investigate the combined effects of dithering and the survey grid geometry on the completeness of coverage provided by the survey. Section 5 discusses miscellaneous details of the application and implementation of dithering. Section 6 summarizes the results.

\section{Evaluation of Dithering Strategies}

\subsection{Dithering}

To be specific, we define the process of "dithering" as obtaining multiple mostly overlapping images of a single field. Normally, each of the dithered images has a different spatial offset from the center of the field, and none of the offsets of the dither pattern is larger than about half of the size of the detector array. Generally, the set of dithered images is averaged in some manner into a single high-quality image for scientific analysis. This is distinct from the processes of "surveying" or "mapping", in which a field much larger than the size of the array is observed, using images that are only partially overlapping. If survey data is combined into a single image for analysis, then the process required is one of mosaicking more than averaging. A region may be surveyed or mapped using dithered images at each of the survey grid points.

There are several reasons why an observer might wish to collect dithered data. One is simply to make sure that no point in the field remains unobserved because it happened to be targeted by a defective pixel in the detector array. To meet this objective, two dither images would suffice, provided their offsets are selected to prevent two different bad pixels from targeting the same sky location. A second reason to dither is so that point sources sample many different subpixel locations or phases. Such a data set allows recovery of higher resolution in the event that the detector pixel scale undersamples the instrumental point spread function. Several procedures have been developed for this type of analysis, which is commonly applied to HST imaging data and 2MASS data (e.g. Fruchter \& Hook 1998; Williams et al. 1996; Lauer 1999; Cutri et al. 1999). A third reason to dither is to obtain a data set which contains sufficient information to derive the detector calibration and the sky intensities from the dithered data alone. As discussed in the 
introduction, for the flat sky approach, the flatness of the background is a more important concern than the particular dither pattern. However, this is reversed when the least squares solution to the calibration is derived (Fixsen et al. 2000). The structure of the sky is less important than the dither pattern which needs to be chosen carefully so that the solution is well-constrained.

In an attempt to cover as wide a field as possible, the detector array often undersamples the instrument point spread function. This undersampling can lead to increased noise in the least squares calibration procedure. There are several ways this extra noise can be alleviated. One way is to use strictly integer-pixel offsets in the dither pattern. However, this requires very precise instrument control, and eliminates the possibility of reconstruction of the image at subpixel resolution (i.e. resolution closer to that of the point spread function). A second way to reduce noise is to assign lower weights to data where steep intensity gradients are present. A third way of dealing with the effects of undersampled data is to use subpixel interlacing of the sky pixels within the least squares solution procedure. This technique may require additional dithering over the region since the interlaced sky subpixels are covered less densely than full size pixels. A fourth means is that the least squares procedure of Fixsen et al. (2000) could be modified to account for each datum $\left(D^{i}\right)$ arising from a combination of several pixel (or subpixel) sky intensities $\left(S^{\alpha}\right)$. This is a significant complication of the procedure.

After the least squares method is used to derived the detector calibration, users can always apply the method of their choice (e.g. "drizzle" described by Fruchter \& Hook 1998) for mapping the set of calibrated images into a single subpixelized image. Such methods may or may not allow continued tracking of the uncertainties and their correlations that the least squares procedure provides.

Dithering involves repointing the telescope or instrument, and thus may require additional time compared to simply taking multiple exposures of the same field. Multiple exposures of the same field without dithering would allow rejection of data affected by transient effects (e.g. cosmic rays), and improved sensitivity through averaging exposures, but of course lack the benefits described above. Whether the time gained by not dithering outweighs the benefits lost, will depend on the instrument and the observer's scientific goals.

\subsection{A Figure of Merit}

The accuracy of the calibration of an array detector cannot be fully specified by a single number or even a single number per detector pixel. The full covariance matrix is necessary to provide a complete description of the uncertainties. The magnitude of the diagonal elements of the covariance matrix (i.e.

$\sigma_{p}^{2}$ ) is determined primarily by the noise characteristics of the instrument and the sky, and is sensitive to the number of images collected in a set of dithered data, but not to the dither pattern. The off-diagonal elements of the covariance matrix are sensitive to the dither pattern, and through the correlations they represent, any measurements made from the calibrated data will contain some imprint of the dither pattern. (In general these correlations degrade the signal quality although they can improve the results of some types of measurements depending on whether the correlations are positive or negative and whether the two data elements are used with the same or opposite sign in the measurement.) In order to obtain the best calibration, one would like to use a dither pattern that minimizes the correlations it leaves in the calibrated data. Since comparison of the entire covariance matrices for different dither patterns is awkward, we adopt a single number, a "figure of merit", that is intended to provide a generic measure of the relative size of the off diagonal terms of the covariance matrix. The figure of merit (FOM) is designed only to compare 
different dither patterns rather than investigating all of the details of a full observing system (i.e. particular telescope/instrument combinations). The instrumental details matter of course, and in practice they may place additional constraints in choosing the dither pattern.

Here we make several simplifying assumptions to ease the calculations and comparisons. First we assume that all of the detector pixels have approximately the same noise and gain. Next we assume that the noise is independent of sky position, either because the Poisson counting statistics are not important or the observed field is so uniform that the photon counting statistics do not vary appreciably across the field. With these assumptions we can simultaneously solve for both the gain and/or offset for each detector pixel and the sky brightness of each sky pixel (Fixsen et al. 2000). The solution necessarily introduces correlations into the uncertainties.

For the figure of merit we choose only a single pixel at the center of the array and look at its correlations. This is done to reduce the calculational burden which includes 4 billion correlations for a modest $256 \times 256$ detector. Since all of the pixels are locked to the same dither pattern the correlations are similar for the other pixels (discussed below). We sum the absolute value of the correlations between the central pixel and all of the other pixels. This is compared with the variance of the central pixel, $\sigma_{p_{0}}^{2}$, as this is the irreducible uncertainty due to detector noise alone. Thus, we define the figure of merit $(F O M)$ as:

$$
F O M=\frac{\sigma_{p_{0}}^{2}}{\sum_{i \in \text { all pixels }}\left|V_{i p_{0}}\right|}
$$

where $V$ is the covariance matrix of the detector parameters. The absolute value is used here to ensure that the sum will be small only if all of the terms are small, not because some of the frequent negative correlations happen to cancel the positive correlations. In detail, the FOM is a function $(f(x) \approx 1 /(1+x))$ of the mean absolute value of the normalized off-diagonal elements of the covariance matrix. With this definition, the FOM is bounded on the range $[0,1]$, and can be thought of as an efficiency of encoding correlations in the dither pattern, i.e. a high FOM is desired in a dither pattern.

Equation 6 is not unique. A wide variety of possible quantitative figures of merit could be calculated. Ideally one would choose the FOM that gives the lowest uncertainties in the final answer. This can be done if the question, i.e. quantity to be measured or scientific goal, is well determined. In that case the question can be posed as a vector (or if there is a set of questions, a corresponding set of vectors in the form of a matrix). The vector (or matrix) can then be dotted on either side of the covariance matrix and the resulting uncertainty minimized. There are several problems in this approach. One is that the matrix is too large to practically fit in most computers. A second problem is that the question may not be known before the data are collected. A third problem is that the same data may be used to answer several questions. To deal with the first issue we use only a single row or column of the symmetric covariance matrix. As shown below, the rows of the matrix have a similar structure over most of the array. To deal with the other two issues, the FOM uses the sum the absolute value of all of the terms. This may not be the ideal FOM for a specific measurement, but it should be a good FOM for a wide variety of measurements to be made from the data.

Throughout this paper, we calculate the FOM based on calibration which only seeks to determine the detector gains or offsets, but not both. When both gains and offsets are sought, the solution for the covariance matrix contains degeneracies that are only broken by the presence of a non-uniform sky brightness (Fixsen et al. 2000). The FOM when solving for one detector parameter is similar to that which would apply when solving for both gains and offsets. 

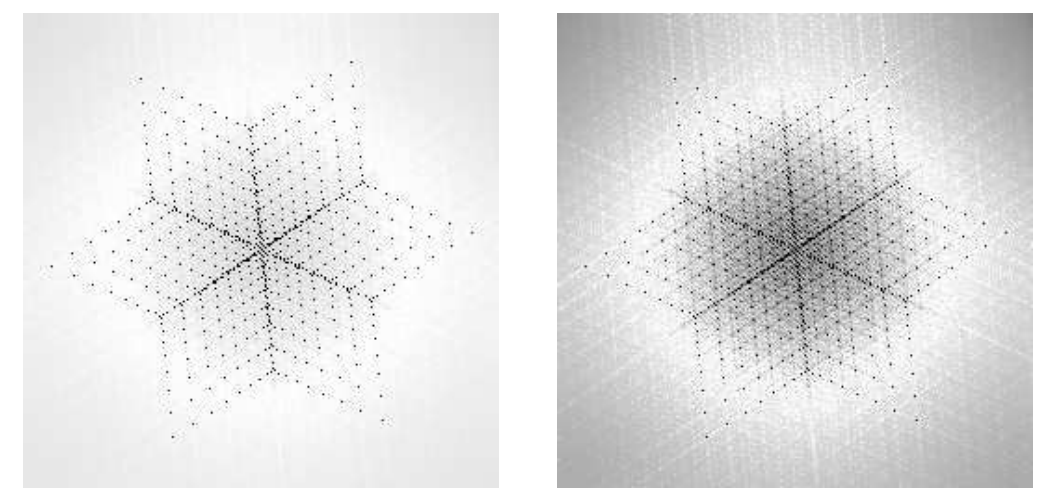

Fig. 1.- On the left is the $u-v$ baseline coverage of the VLA for a snapshot of a source at the zenith. In the center and on the right is the map of $\left|V_{i p_{0}}\right|$ for a "VLA" dither pattern, stretched to emphasize the similarity to the VLA $u-v$ plane coverage, and the weaker correlations respectively.

\subsection{Dither Patterns and Radio Interferometers}

In order to compute relative gain and/or offset, two detector pixels must observe the same sky pixel or have a connection through other detector pixels that mutually observe one or more sky pixels. A shorter path of intermediate detectors implies a tighter connection and lower uncertainties. One goal of dithering is to tighten the connections between detectors and thus lower the uncertainties. This combinatorial problem happens to share geometrical similarities with another problem that has been dealt with previously, namely covering the $u-v$ plane with a limited number of antennas in a radio interferometer.

Figure 1 shows the $u-v$ coverage of the VLA for a snapshot of a source at the zenith. Each antenna pair leads to a single sample marked with a dot in the $u-v$ plane. Also shown is the map of $\left|V_{i p_{0}}\right|$ generated by using a 27-position dither pattern with the same geometry as the VLA array ( $\$ 3.2)$. The strongest correlations are found at locations of the direct dither steps corresponding to the VLA baselines. However, the non-zero correlations (and anti-correlations) found elsewhere in the map make a significant contribution to the total FOM.

Figure 2 shows maps of $\left|V_{i p_{0}}\right|$ generated using different choices of $p_{0}$. These maps illustrate that the correlations of all pixels are similar in structure to those of the central pixel, but the finite size of the detector limits the correlations available to pixels near the detector edges. The dither pattern used in this demonstration is the VLA pattern described in $\S 3.2$.

Despite the similar geometries of radio interferometer $u-v$ coverage and dither pattern maps of $\left|V_{i p_{0}}\right|$, several important differences should be noted. First, with radio telescopes only direct pairs of antennas (although all pairs) can be used to generate interference patterns, whereas with dither patterns a path involving several intermediate detector pixels can be used to generate an indirect correlation. However, the greater the number of intermediate steps that must be used to establish a correlation, the noisier it will be. Second, the $u-v$ coverage is derived instantly. Observing over a period of time fills in more of the $u-v$ plane as the earth's rotation changes the interferometer baselines relative to the target source. In contrast, the $\left|V_{i p_{0}}\right|$ coverage shown in Figs. 1 and 2 is only achieved after collecting dozens of dithered images. To fill in additional coverage, the dither pattern must be altered directly because there is no equivalent of the earth rotation that alters the geometry of the instrument with respect to the sky. Another important difference is that the short interferometer baselines (found near the center of the $u-v$ plane) are sensitive 

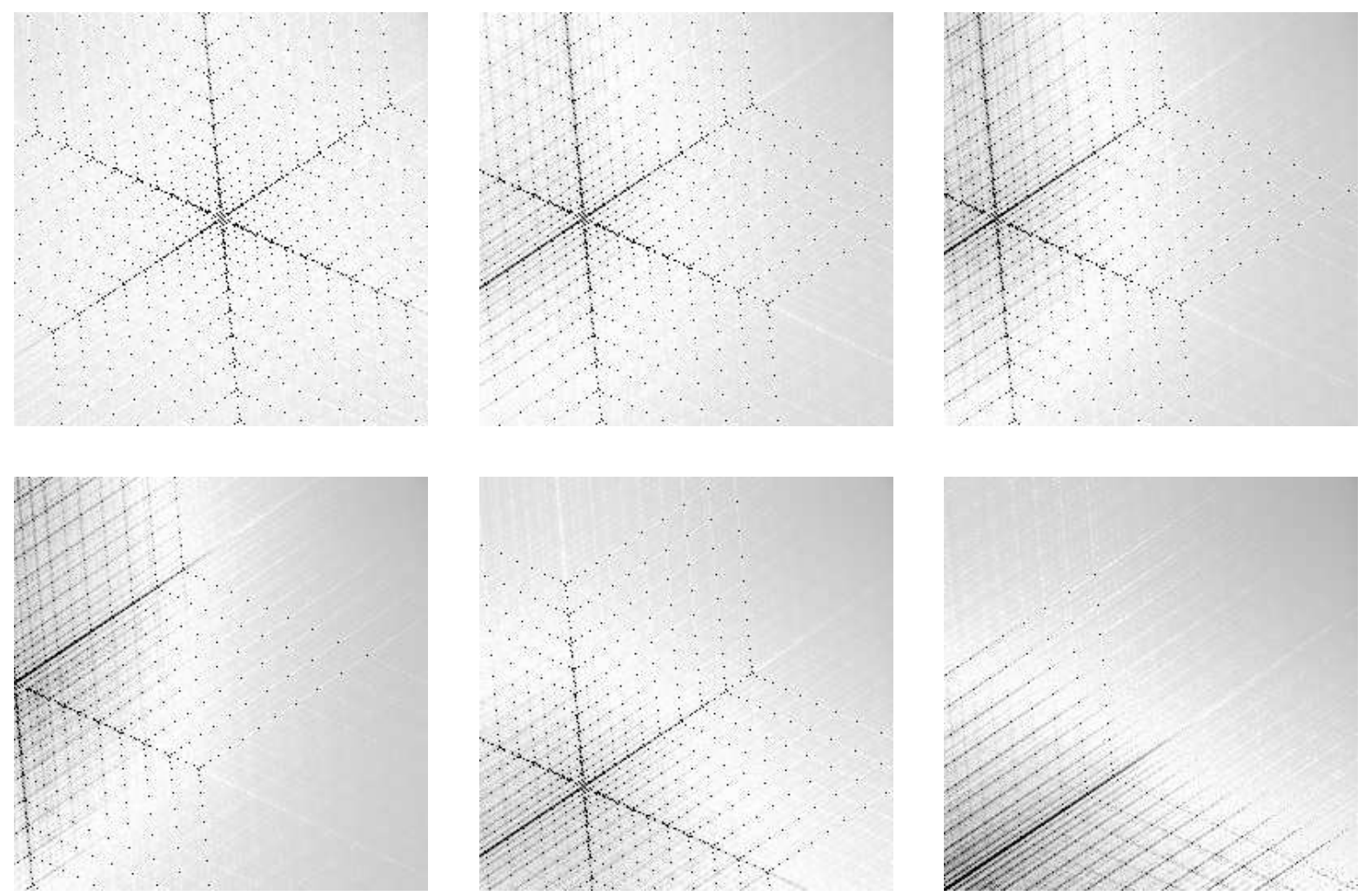

Fig. 2.- The panels show the $\left|V_{i p_{0}}\right|$ correlations for detectors at the locations $(128,128),(64,128),(32,128)$, $(0,128),(64,64)$, and $(0,0)$ in a $256 \times 256$ array (left to right and top to bottom). Dark spots represent strong correlations. The dither pattern used to calculate these correlations is a 27-point VLA pattern.

to the large-scale emission. For dither patterns the inverse relation holds. Direct correlations between nearby detector pixels are sensitive to small-scale structure in the detector properties and sky intensities. Thus the outer edge of the interferometer's $u-v$ coverage represents a limit on the smallest-scale structure that can be resolved, while the outer edge of strong $\left|V_{i p_{0}}\right|$ correlations represents a limit on the largest-scale variations that can be reliably distinguished.

Overall, the geometrical similarities suggest that patterns used and proposed for radio interferometers may prove to be a useful basis set for constructing dither patterns. In the following section, we calculate the FOM for several patterns inspired by radio interferometers in addition to other designs.

\section{Various Dither Patterns}

Several general algorithms for generating dither patterns have been examined. In many cases, we have also explored variants of the basic algorithms by changing functional forms, adding random perturbations, or applying overall scale factors. We have also tested several specific examples of dither patterns from various sources. Examples of the patterns described below are shown in Figure 3. All tests reported here assumed detector dimensions of $256 \times 256$ pixels unless otherwise noted. 


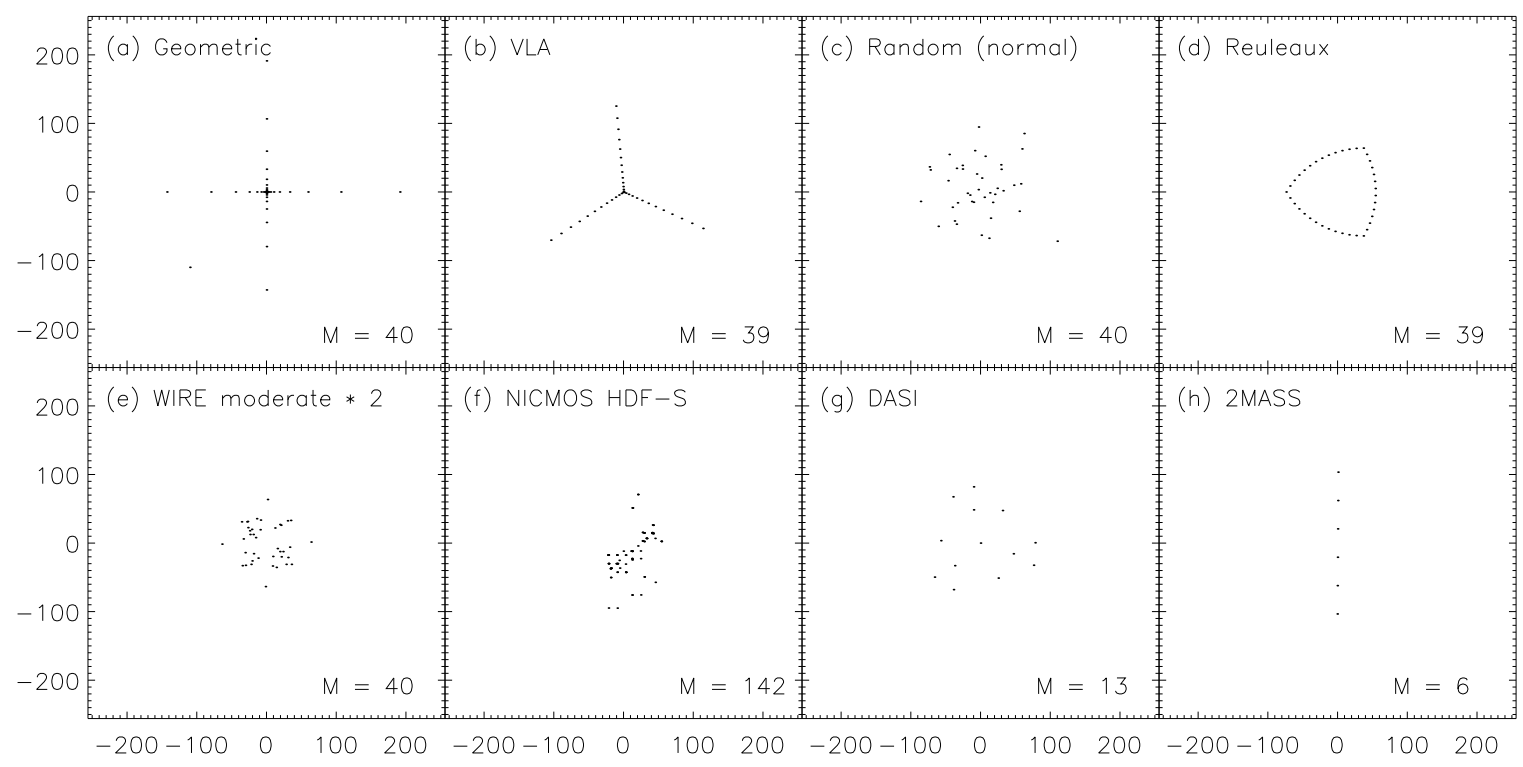

Fig. 3.- Examples of some of the tested dither patterns. The dots mark the center of the array for each of the $M$ positions for each pattern.

\subsection{Reuleaux Triangle}

Take an equilateral triangle and draw three $60^{\circ}$ arcs connecting each pair of vertexes, while centered on the opposite vertex. The resulting fat triangle is a Reuleaux triangle. This basic shape has been used to set the geometry of the Sub-Millimeter Array (SMA) on Mauna Kea (Keto 1997).

This shape can be used as a dither pattern by taking equally spaced steps along each side of the Reuleaux triangle. The length of the steps is set by the overall size of the triangle (a free parameter) and the number of frames to be used in the pattern. For an interferometer, Keto (1997) shows that the $u-v$ coverage can be improved by displacing the antennas from their equally spaced positions around the triangle.

\subsection{VLA}

The "Y"-shaped array configurations of the Very Large Array (VLA) radio interferometer are designed such that the antenna positions from the center of the array are proportional to $i^{1.716}$ (Thompson, et al. 1980). The three arms of the array are separated from each other by $\sim 120^{\circ}$. We have adopted this geometry to provide a dither pattern with positions chosen along each of the three arms at

$$
d r=\sqrt{d x^{2}+d y^{2}}=i^{p} \text { where } i=1,2,3, \ldots, M / 3 .
$$

and $p$ is an arbitrary power which can be used to scale the overall size of the pattern. The first step along each of the 3 arms is always at $d r=1.0$. The azimuths of the arms were chosen to match those of the VLA, at $355^{\circ}, 115^{\circ}$, and $236^{\circ}$. 


\subsection{Random}

Random dither patterns were tested using $d x$ and $d y$ steps generated independently from normal (Gaussian) or from uniform (flat) distributions. The widths of the normal distribution or the symmetric minimum and maximum of the uniform distribution are free parameters.

\subsection{Geometric Progression}

We have generated a geometric progression pattern, stepping in $x$ in steps of $(-f)^{n}$, where $n=0,1, \ldots N-1$ and $f^{N}=256$. The same steps are also used in the $y$ direction. This pattern separates the $x$ and $y$ dimensions. In each dimension the pattern is quite economical in generating correlations up to the point where $f=2$. Beyond this there is little to be gained in adding more dither steps in the $x$ or $y$ direction. However, there is some benefit expected in adding steps combining $x$ and $y$ offsets. Hence, for a $256 \times 256$ array, we should expect the geometric pattern to be good for $M \leq 2 \log _{2}(256)=16$ positions and not show much improvement by adding more positions.

The geometric progression patterns used here contain two additional steps chosen at $(d x, d y)=(0,0)$ and at a position such that $\sum d x=\sum d y=0.0$. This is a cross-shaped pattern, with one diagonal pointing, from which any desired pixel-to-pixel correlation can be made with a small number of intermediate steps. The alternating sign of the steps builds up longer separations quickly.

\subsection{Other Patterns}

Several other patterns were also tested with little or no modifications. The patterns that were planned for the WIRE moderate and deep surveys were examined with both the nominal dither steps, and with steps scaled by a factor of 2 to account for the difference between the $128 \times 128$ pixel WIRE detectors and a larger $256 \times 256$ pixel detector. The pattern used for NICMOS observations of the HDF-S was tested. The configuration of the 13 antennas of the Degree Angular Scale Interferometry (DASI; Halverson, et al. 1998) was used as a scalable pattern. The declination scanning employed by 2MASS yields a linear dither pattern.

\subsection{Figures of Merit for the Patterns}

In the simplest form, a specific pattern, $M$ images deep, would be used to collect data at a single target. The FOM for all patterns tested, with various $M$ and other modifications, are listed in Table 2. For all patterns, the FOM increases (improves) as $M$ increases. For $M<20$ the change is quite rapid. The variations of FOM as a function of $M$ for the tabulated versions of each of the patterns are shown in Figure 4 .

Table 2 also lists results for a Reuleaux triangle pattern applied to a $32 \times 32$ detector, and for two large grid dither patterns applied to the same array. The grid dither patterns are square grids with 1 pixel spacings between dithers, such that for the $M=1024$ pattern a single sky pixel is observed with each detector, and for the $M=4096$ pattern a $32 \times 32$ pixel region of sky is observed with each detector

pixel. These results demonstrate that in the extreme limit where all correlations are directly measured, the $F O M \rightarrow 1.0$. The FOM does not reach 1.0 because of the finite detector and dither pattern sizes. 

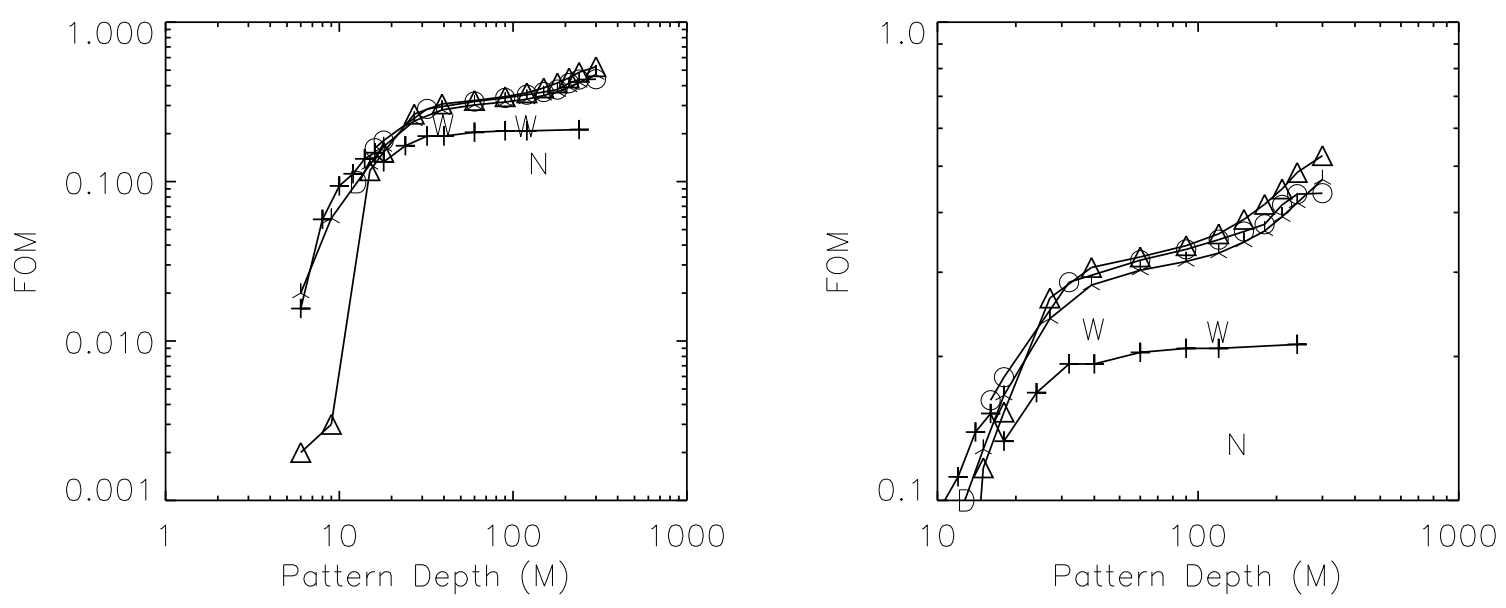

Fig. 4.- The FOM as a function $M$ the number of positions in each pattern. "+" = geometric progression, inverted "Y" = VLA, "O" = random (normal), " $\triangle "$ = Reuleaux triangle, "W" = WIRE moderate and deep surveys, "D" = DASI, and "N" = NICMOS coverage of the HDF-S. The right panel shows the same data on an enlarged scale.

For the $256 \times 256$ arrays, the Reuleaux and random (normal) patterns have the best FOM for $M>20$. The VLA pattern is only a little worse, but other patterns have distinctly smaller FOM than these patterns. For the scalable VLA, random, Reuleaux, and DASI patterns, the best FOM for a fixed $M$ usually occurs when the maximum $|d x|$ or $|d y| \approx 128$ pixels. For patterns with small $M$ the optimum scale factor is usually smaller, to avoid too many large spacings between widely scattered dither positions. For values of $M<20$ no pattern seems to produce a good FOM, however, the geometric pattern usually does best in this regime. Rotating the patterns with respect to the detector array generally produces only modest changes in the FOM. For $M \lesssim 30$, the FOM of a Reuleaux pattern is improved by adding small random perturbations to the dither positions. No optimization of the perturbations was performed (as Keto 1997), but apparently any perturbation is better than none for small $M$ patterns. Deep Reuleaux triangle patterns are neither improved nor worsened by small perturbations.

The results presented in Fig. 4 and Table 2 indicate that a good FOM is dependent on patterns that sample a large number and wide range of spatial scales. A variety of patterns with different geometries can yield satisfactory results, as demonstrated by the rather different Reuleaux triangle and random patterns. Therefore, attempts to find the single "optimum" pattern may not be very useful, and selection of a dither pattern needs to carefully avoid patterns that contain obvious or hidden redundancies that lead to a poor FOM. An example of this sort of pitfall is the $M=18$ geometric pattern, for which all dither steps are integer powers of 2, leading to a FOM that is worse than geometric patterns with depths of $M=14$ or 16 .

The coverage of the VLA, random, and Reuleaux triangle dither patterns when used for observation of a single target is shown as maps in Figure 5, and histograms in Figure 6. The Reuleaux triangle dither pattern provides the largest region covered at maximum depth, but if a depth less than the maximum is still useful then the VLA dither pattern may provide the largest area covered.

The importance of the largest dither steps in a pattern is demonstrated through analysis of simulated 

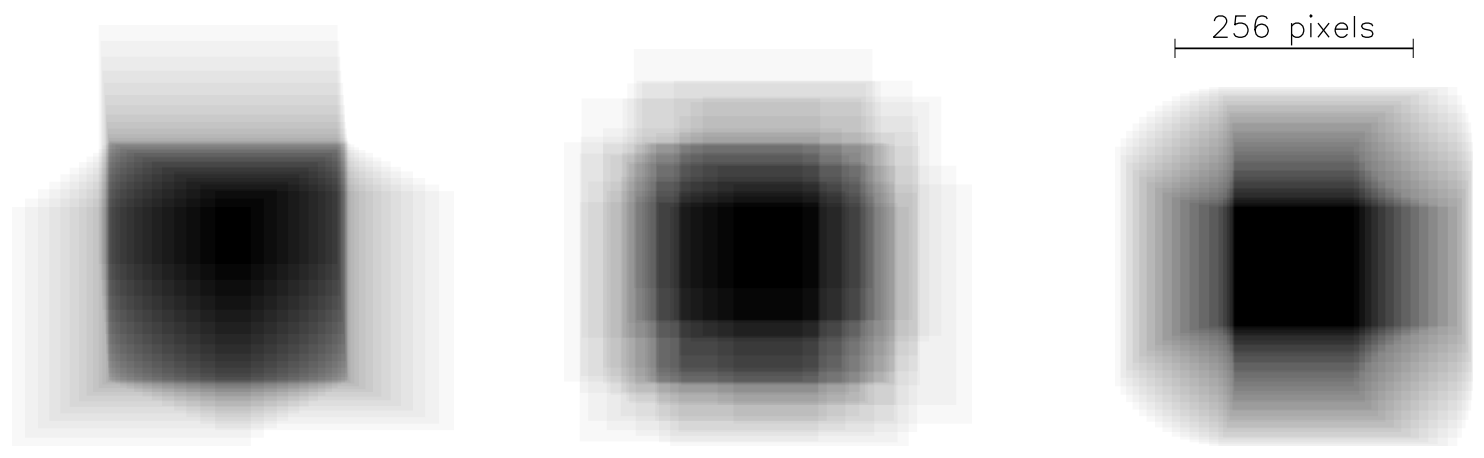

Fig. 5.- Coverage maps for $M=39$ single target dither patterns (left) VLA: $F O M=0.282$, (center) Random Gaussian: $F O M=0.302$, (right) Reuleaux triangle: $F O M=0.307$.
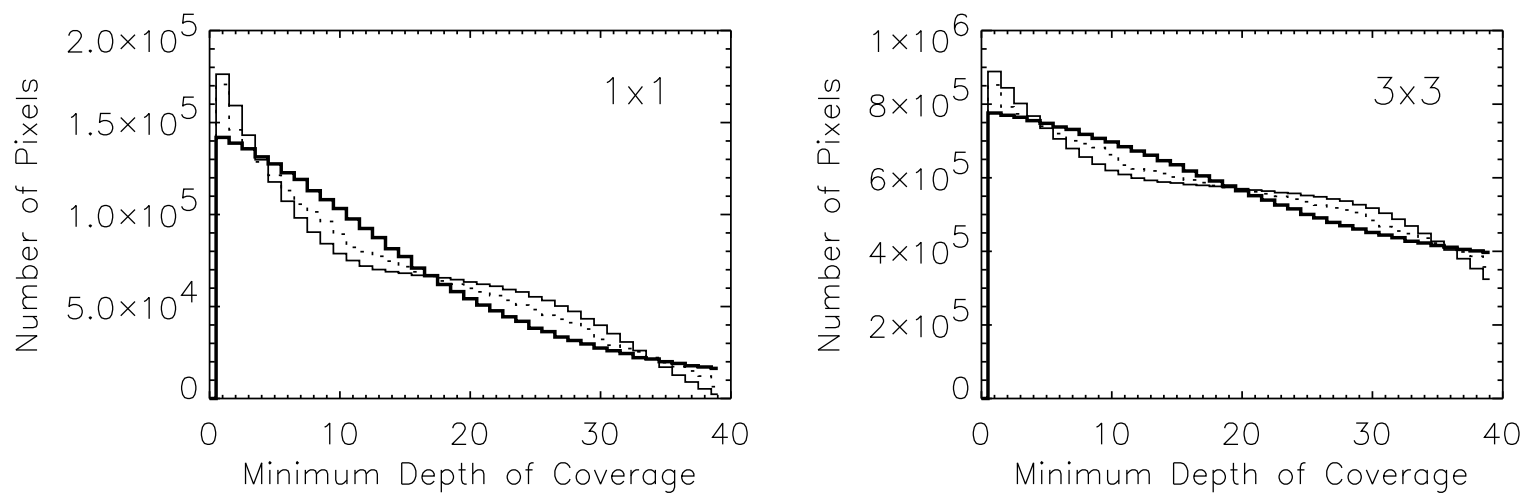

Fig. 6.- Cumulative histograms of the coverage as a function of minimum depth for $M=39$ VLA (thin), random Gaussian (dotted), and Reuleaux triangle (thick) dither patterns. Coverage for a single target is shown at left; coverage for a deep $3 \times 3$ survey with a $256 \times 256$ pixel grid spacing is shown at right.

WIRE data. A synthetic sky was sampled using both geometric progression and random dither patterns. The maximum dither offset was 38 pixels for the geometric progression pattern and 17 pixels for the random pattern. The FOM for this geometric pattern is 0.127 , and for this random pattern it is 0.099. WIRE's detectors were 128 arrays. The gain response map used in the simulations contained large scale gradients with amplitudes of $\sim 10 \%$. Figure 7 shows comparisons between the actual gains and the gains derived when the self-calibration procedure described by Fixsen, et al. (2000) is employed. The random dither pattern without the larger dither offsets was less effective at identifying the large scale gain gradient. The undetected structure in the gain winds up appearing as a sky gradient that affects the photometry of both the point sources and the background in the images.

\section{Surveys}




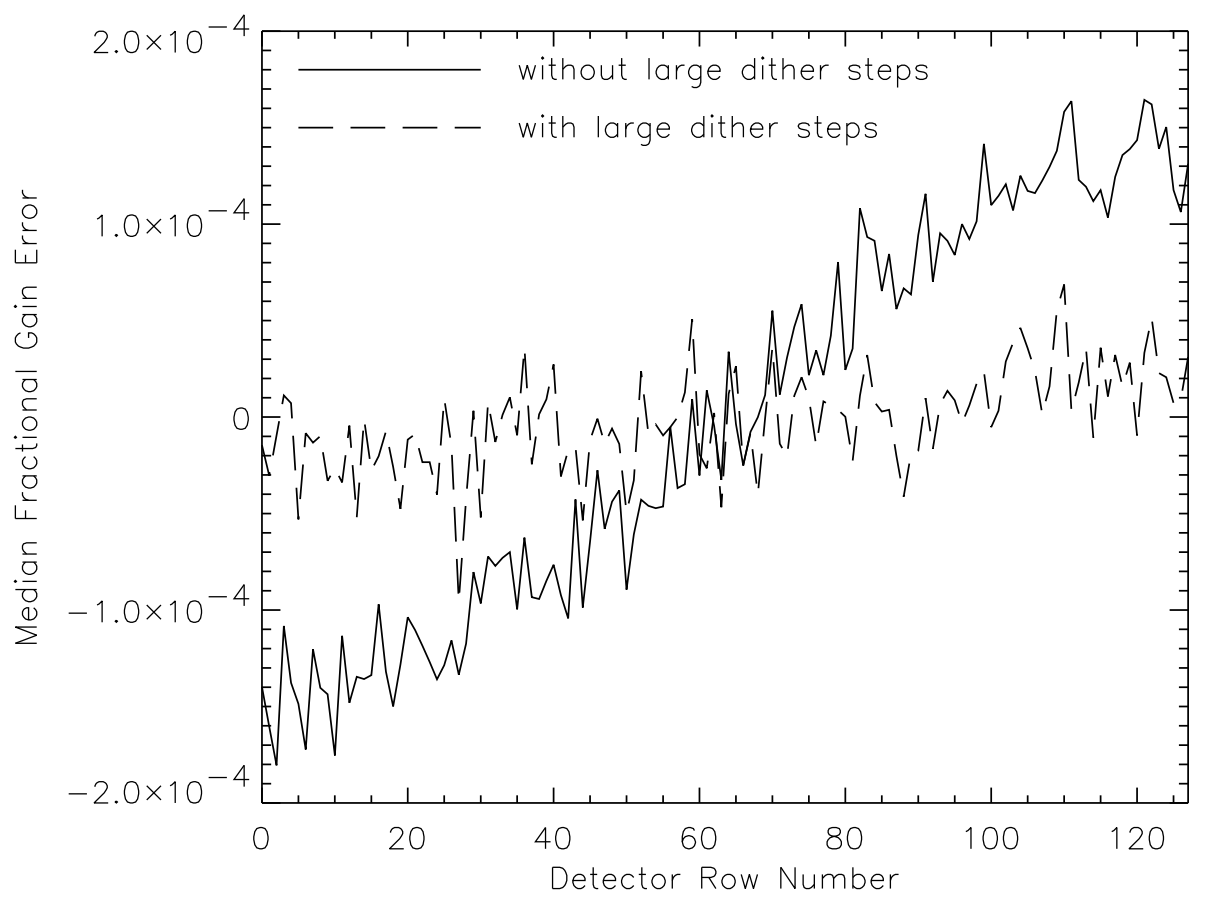

Fig. 7.- The median fractional gain errors are plotted as a function of detector row for detector gains derived from two simulated WIRE data sets. Each simulation contains 10 dithered images. Only one simulation includes relatively large dither steps. When applying a self-calibration algorithm, a lack of large dither steps leads to large-scale gain errors.

\subsection{Deep Surveys}

For obtaining a standard deep survey, we have assumed that the same dither pattern is repeated at each location of a grid. The survey grid is assumed to be aligned with the detector array and square, with a spacing no larger than the size of the array. The FOM for surveys using several different dither patterns and grid spacings are listed in Table 3. The FOM derived for the entire survey as a single data set is basically determined by the FOM of the dither pattern used. The overlap between dithers from adjacent points in the survey grid, effectively adds additional steps to the dither pattern, which slightly improves the FOM over that of the pattern when used for a single target. Smaller survey grid spacings lead to increased overlap and increased FOM, but also lead to a smaller area of sky covered in a fixed number of frames. The improvement in the FOM when used in surveys rather than singly is most significant for relatively shallow dither patterns, however, even in a survey, the FOM of a shallow pattern is still not very good. The FOM improves only slightly as the survey grid grows larger than the basic $2 \times 2$ unit cell. 


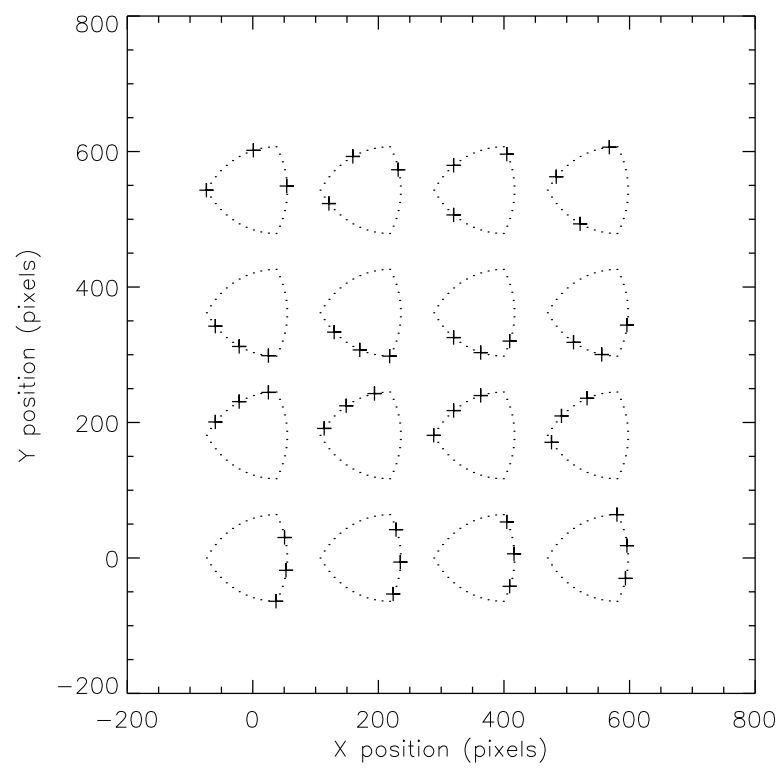

Fig. 8.- An example of a $4 \times 4 M=3$ shallow survey on a $181 \times 181$ pixel grid using an $M=33$ Reuleaux triangle dither pattern. The dots show the repetition of the full dither pattern, while the crosses mark the dither points that were actually used at each survey grid point.

\subsection{Shallow Surveys}

For shallow surveys in which as few as 2 images per grid location are desired, using the same small $M$ dither pattern at each location yields a very poor FOM. An alternate method of performing a shallow survey is to choose a larger $M$ dither pattern and apply successive steps of the dither pattern at successive locations in the survey grid (Figure 8). If the survey is large enough, it can contain all the direct correlations of the large $M$ dither pattern, though spread out among many survey grid points rather than at a single location. The FOM of the shallow survey can thus approach the FOM of the single deeper dither pattern. The advantage of altering the dither pattern at each survey grid point is still present, though less significant, as the survey depth increases. The FOM derived from various surveys using this shallow survey strategy are shown in Table 4.

A random dither pattern is a natural choice for use in this shallow survey strategy. One can proceed by simply generating a new random set of dithers at each survey grid point. If a more structured dither pattern is used as the basis for the shallow survey (e.g. the Reuleaux triangle in Fig. 8), then one must address the combinatorial problem of selecting the appropriate subsets of the larger dither pattern at each survey grid point. The example shown in Fig. 8 is not an optimized solution to the combinatorial problem.

\subsection{Survey Coverage \& Grids}

When a large area is to be observed, the most efficient way to cover the region is to use a square survey grid aligned with the detector array and with a grid spacing equal to the size of the array, or slightly less to 
guard against bad edges or pointing errors. In this mode a deep survey using the same $M$ position dither pattern at each survey grid point will cover the desired region at a depth of $M$ or greater. There will be no holes in the coverage, though the edges of the surveyed region will fade from coverage of $M$ to 0 with a profile determined by the dither pattern used (Fig. 6). A shallow survey, using a different dither pattern at each grid point, may or may not have coverage holes depending on the maximum size of the dither steps and the grid spacing of the survey. The constraint for avoiding coverage holes is that the overlap of the survey grid must be more than the maximum range of dither step offsets (independently in the $x$ and $y$ coordinates), e.g.

$$
X-\Delta X>\max \left(d x_{i}\right)-\min \left(d x_{i}\right)
$$

where $X$ is the size of the array, $\Delta X$ is the survey grid spacing, and $d x_{i}$ are the dither steps $(i=1 \ldots M)$. This constraint places the survey grid points close enough together that coverage holes are avoided even if dithers at adjacent grid point are offset in the maximum possible opposite directions. If the shallow survey observing program can be arranged to avoid this worst case, then the grid spacing may be increased without developing coverage holes. Coverage holes may be undesirable when mapping an extended object, but may be irrelevant if one is simply seeking a random selection of point sources to count. Note that some minor coverage holes are inevitable, where data are lost to bad pixels or cosmic rays. Additionally, a coverage hole where a depth of $M=1$ is achieved instead of $M=3$ might be more serious than one where $M=18$ is achieved instead of $M=20$.

For this shallow survey strategy there is an inherent tradeoff between the area covered (without holes) and the FOM. Using a dither pattern containing large dither steps as the basis for the survey will lead to a good FOM, but require a relatively large overlap in the survey grid spacing and a consequent loss of area covered by the survey. Decreasing the scale of the dither pattern leads to a lower FOM, but permits an increase in the survey grid spacing and total area covered. The ideal balance between these will depend on the instrumental characteristics and the scientific objectives.

In many instances, an observer may want to survey or map a region of fixed celestial coordinates. In some cases, instrumental constraints (i.e. the ability to rotate the telescope or detector array relative to the optical boresight) may not allow alignment between the detector array and the desired survey grid. This will result in coverage holes in the surveyed region, unless the grid spacing is reduced enough to prevent holes regardless of the array orientation. If a square grid with a spacing of $\Delta X=X / \sqrt{2}$ is used then coverage holes are prevented for any possible orientation of the arrays. This is illustrated by plots in the first two rows of Figure 9, which shows the array positions for $4 \times 4 M=1$ survey (without dithering). With a deep survey strategy, avoidance of holes in the $M=1$ case will prevent holes at any depth $M$, but for the shallow survey strategy additional overlap may need to be built into the survey grid to prevent holes as discussed above. Decreasing the survey grid by a factor of $\sqrt{2}$ in each dimension results in a grid that covers only half the area that could be covered if the detectors and grid are aligned. This efficiency can be increased if the survey is set up on a triangular grid rather than a square grid. If alternate rows of the survey grid are staggered by $X / 2$ (middle row of Fig. 9) and the vertical spacing of the grid is reduced by a factor of $\sqrt{3} / 2$, then holes are prevented as long as the array orientation remains fixed throughout the survey (4th row of Fig. 9). The area covered by this triangular grid will be $\sim 87 \%$ of the maximum possible area, rather than $50 \%$ for the square grid required to prevent holes. If the array orientation is not fixed throughout the survey (last column of Fig. 9) then the triangular grid must be reduced by an additional factor of $\sqrt{3} / 2$ in both dimensions. This results in a $\sim 65 \%$ efficiency for the triangular grid versus $50 \%$ for the square grid, which requires no further reduction. The FOM of a survey on a triangular grid is similar to that of a survey on a square grid with an equivalent amount of overlap. 


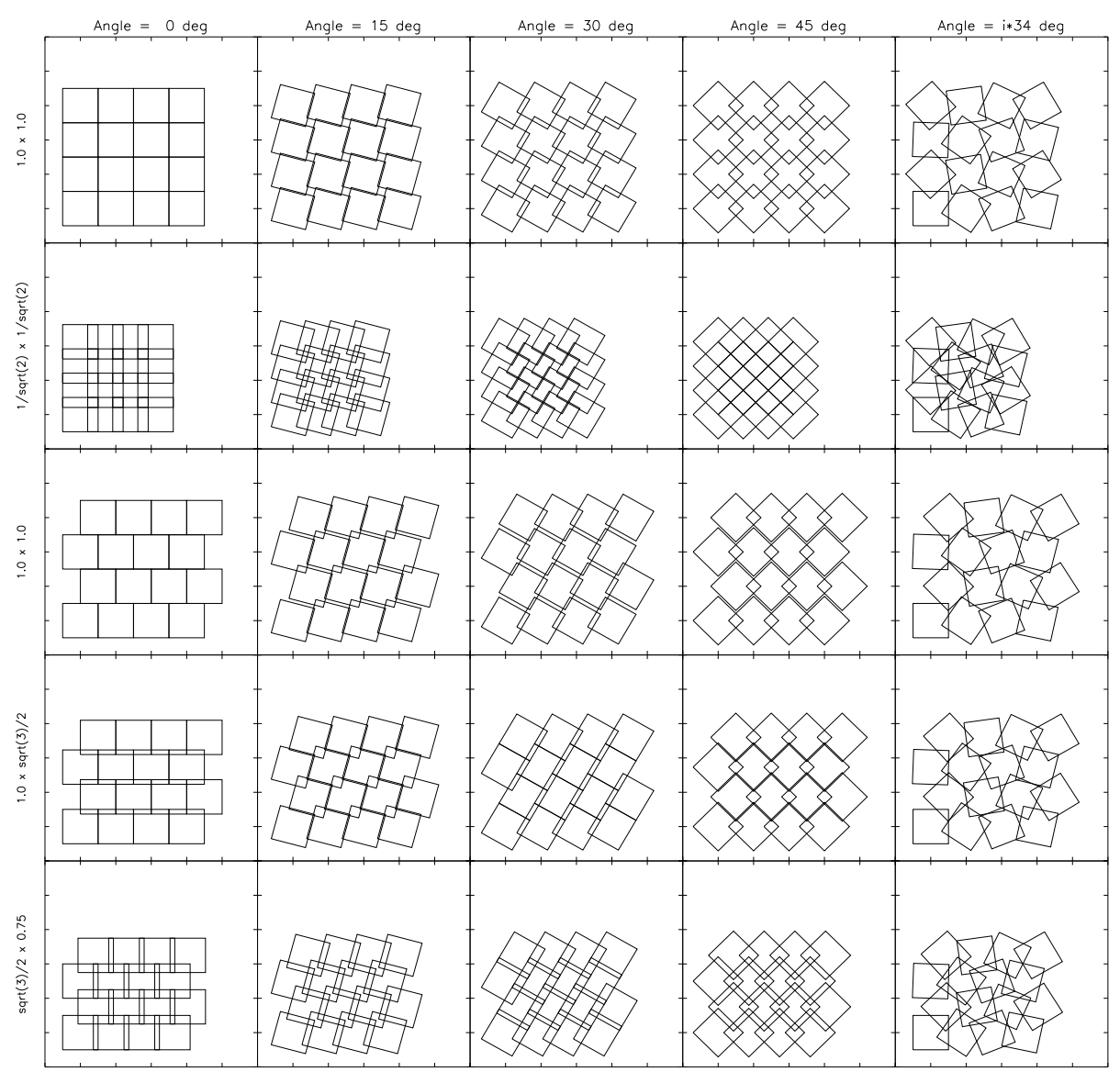

Fig. 9. - Examples of $4 \times 4 M=1$ surveys on square (1st and 2nd rows), staggered (middle row), and triangular grids (4th and 5th rows) for various angles between the detector array and the grid orientation. In the last column the array orientation was rotated by $34^{\circ}$ at each successive survey grid point. The squares indicate the outline of the entire array as pointed at each survey grid point.

\section{Other Miscellaneous Details}

The most flexible implementation of the dithering strategies presented here would be to have the dither steps be determined algorithmically from a small set of user-supplied parameters. For example, an observer could select: a type of dither pattern (e.g. Reuleaux triangle or random), a pattern depth $M_{\text {pattern }}$, and a scaling factor to control the overall size of the pattern. From this information, the telescope control software could calculate and execute the desired dither pattern. For the shallow survey strategy presented above, the observer would also need to supply: the survey depth, $M_{\text {survey }}<M_{\text {pattern }}$, and perhaps an index to track which grid point of the survey is being considered (software might handle this automatically).

Sometimes design or operational constraints require that the dither patterns reside in a set of pre-calculated look-up tables. In this case (which has applied to both WIRE and IRAC) the observer's ability to set the dither pattern is more limited. However, some of the limitations of using dither tables 
can be mitigated if the observer is not forced to use dither steps from the tables in a strictly sequential fashion. For example, one dither table might contain an $M=72$ Reuleaux triangle dither pattern calculated on a scale to produce the optimum FOM. If the observer is allowed to set the increment, $\Delta i$, used in stepping through this dither table, then by selecting $\Delta i=3$ or $\Delta i=4$, then dither patterns of $M=24$ or $M=18$ can be generated. Allowing non-integer increments (subsequently rounded) would enable the selection of a dither pattern of any depth $M \leq 72$. This adjustment of the increment is most clearly useful for very symmetric dither patterns such as the Reuleaux triangle pattern. For a dither table containing a random pattern, non-sequential access to the table can have other uses. First, in applying the shallow survey strategy, a random dither table of length $M_{\text {pattern }}$ could be used to sequentially generate $M_{\text {survey }}<M_{\text {pattern }}$ dithers at each successive survey grid point. Selection of dither steps would wrap around to the beginning of the table once the end of the table is reached. For example a dither table of $M_{\text {pattern }}=100$ could be used sequentially to generate 20 different patterns for an $M=5$ shallow survey. Even better would be to have a table with $M_{\text {pattern }}$ a prime number, e.g. 101. Then, wrapping the table allows the sequential generation of $M_{\text {pattern }}$ different dither patterns for any $M_{\text {survey }}$, though some of these dither patterns will differ from others by only one step. Additional random patterns can be generated by setting different increments for stepping through the table. Enabling specification of the starting point in the dither table would additionally allow the observer to pick up the random dither pattern sequence at various (or the same) positions as desired. These capabilities would enable an observer to exploit the large number of combinations of dither steps available in a finite length dither table, in efforts to maximize the FOM. Use of a fixed dither table can also be made less restrictive if a scaling factor can be applied to the dither pattern size. A free scaling factor provides an additional means of adjusting the pattern size as desired to meet coverage or FOM goals.

For the cases presented in this paper, we have assumed that the orientation of the detector array remains fixed throughout the execution of the dither pattern and any larger survey (except for the last column of Fig. 9). However, rotation of the detector array relative to the dither pattern, either within a single pointing, or at different pointings in a deep survey, is an effective way of establishing combinations of direct pixel-to-pixel correlations that cannot be obtained using purely translational dither steps. Inclusion of rotation of the detector can lead to further improvements in the FOM of a given dither pattern or survey. In the extreme, a dither pattern could even be made entirely out of rotational rather than translational dither steps. However, without an orthogonal "radial" dither step, rotation alone is similar to dithering with steps in the $x$-direction but not the $y$-direction. The ability to implement rotations of the detector will be allowed or limited by the design and operating constraints of the telescope and instruments being used.

Bright sources can often saturate detectors and cause residual time-dependent variations in detector properties. For observations of a field containing a bright source, use of a random dither pattern may lead to streaking as the source is trailed back and forth across the detector array between dithers. In contrast the use of a basically hollow or circular dither pattern such as the Reuleaux triangle pattern, will only trail the source through a short well-defined pattern, which will lie toward the outer edge of the detector if the source position is centered in the dither pattern. If the pattern scale of the dither pattern is increased, the trail of the source can be pushed to or off the edges of the detector, though the FOM will suffer if the pattern scale is greatly increased. In other words, a hollow dither pattern with a large scale could be used to obtain a series of images looking around but not at a bright source.

Dithering may be performed by repointing the telescope, or by repositioning the instrument in the focal plane, for example through the use of a tilting optics as in the 2MASS (Kleinmann 1992) or SIRTF MIPS (Heim, et al. 1998) instruments. Calculation of the FOM of the dither pattern will be independent 
of the technique used. The self-calibration procedure, however, may be affected by effective instrumental changes if it is repositioned in the focal plane. The alternative repointing of the telescope can be much more time consuming and may limit the use of large $M$ dither patterns.

The combined use of two or more non-contiguous fields is transparent to the self-calibration procedure. If the same dither pattern is used on each of the separate fields, the resulting FOM will be the same as that for a single field. The FOM would be improved for the combined data set if the dither pattern is different for each of the subset. The FOM for data set of non-contiguous regions is thus similar to that obtained using the same dither strategy in a contiguous survey, except there is a small loss in the FOM because of the lack of overlap between adjacent regions.

Another means of minimizing coverage holes when using a shallow survey strategy is to oversample the depth of the survey. For example, performing the shallow survey at a depth of $M=4$ when $M=3$ is the intended goal will result in fewer holes at a depth of 3 for a fixed grid spacing, and in a better FOM for the overall survey. However, the cost in time of the additional exposures may be prohibitive.

The FOM as calculated here only depends upon the offsets of the dither pattern rounded to the nearest whole pixel. This means that any desired combination of fractional pixel offsets to facilitate subpixel image reconstruction may be added to the dither patterns without affecting the various aspects discussed in this paper. If using dither tables, one could have separate tables for the large scale and the fractional pixel dithers, with the actual dithers made by adding selected entries from the two tables. This could allow simultaneous and independent implementation of large-scale and subpixel dithering strategies. Only subpixel image reconstruction that demands exclusively small ( $\sim 1$ pixel) dithering would be incompatible with the dithering strategies presented here.

\section{Conclusion}

We have shown that proper selection of observing strategies can dramatically improve the quality of self-calibration of imaging detectors. We have established a figure of merit (Eq. 6) for quantitatively ranking different dither patterns, and have identified several patterns that enable good self-calibration of a detector on all spatial scales. The layouts of radio interferometers correspond to good dither patterns. Both the highly ordered Reuleaux triangle pattern and the unstructured random pattern provide good FOM with moderate or deep observations. This indicates that good patterns must sample a range of spatial scales without redundancy, and if this condition is met, then secondary characteristics of the patterns or instrument constraints may determine the actual choice of the dither pattern. Any dither pattern must contain steps as large as half the size of the detector array if large scale correlations are to be effectively encoded in the dithered data set. Deep surveys can take advantage of the use of a single good dither pattern. Shallow surveys can obtain good FOM by altering the dithers used at each of the survey grid points. Using a fixed pattern throughout a shallow survey makes it difficult or impossible to apply a self-calibration procedure to the resulting data sets. The use of triangular instead of square survey grids can be more efficient in executing complete-coverage surveys when the array orientation cannot be set to match the survey grid. Good dither patterns and survey strategies can be devised even in some seemingly restricted situations. The ultimate importance of dithering and a good FOM will depend on the nature of the instrument and the data and on the scientific goals. For many goals, obtaining a larger quantity of data may not be an adequate substitute for obtaining data with a good FOM. 
We thank D. Shupe and the WIRE team for supplying simulated data using several different dither patterns. W. Reach and members of the SSC and IRAC instrument teams were helpful in providing useful ideas and criticism throughout the development of this work. J. Gardner, J. Mather, and the anonymous referee provided very helpful criticism of the manuscript.

\section{REFERENCES}

Cutri, R., Van Dyck, S. \& the 2MASS Team, 1999, Explanatory Supplement to the 2MASS Spring 1999 Incremental Data Release, http://www.ipac.caltech.edu/2mass/releases/spr99/doc/explsup.htm]

Fixsen, D. J., et al. 1994, ApJ, 420, 457

Fixsen, D. J., Moseley, S. H., \& Arendt, R. G. 2000, ApJS, 128, in press

Fruchter, A. S. \& Hook, R. N. 1998, PASP, submitted, (astro-ph/9808087)

Gardner, J. P. 1995, ApJS, 98, 441

Halverson, N. W., Carlstrom, J. E., Dragovan, M., Holzapfel, W. L., \& Kovac, J. 1998, in Advanced Technology MMW, Radio, and Tetrahertz Telescopes, Proc. SPIE 3357, ed. T. G. Phillips (Bellingham, WA: SPIE), 416

Heim, G. B., et al. 1998, in Space Telescopes and Instruments V, Proc. SPIE 3356, ed. P. Y. Bely, \& J. B. Breckinridge, (Bellingham, WA: SPIE), 985

Joyce, R. R. 1992, in ASP Conf. Ser. 23, Astronomical CCD Observing and Reduction Techniques, ed. S. B. Howell, (San Francisco: ASP), 258

Keto, E. 1997, ApJ, 475, 843

Kleinmann, S. G. 1992, in Robotic Telescopes in the 1990s, ed. A. V. Filippenko, (San Francisco: ASP), 203

Lauer, T. R. 1999, PASP, 111, 227

Mather, J. C., et al. 1994, ApJ, 420, 439

Thompson, A. R., Clark, B. G., Wade, C. M., \& Napier, P. J. 1980, ApJS, 44, 151

Tyson, J. A. 1986, J. Opt. Soc. Am. A, 3, 2131

Tyson, J. A. \& Seitzer, P. 1988, ApJ, 335, 552

Williams, R. E., et al. 1996, AJ, 112, 1335 
Table 1. Comparison of Data Reduction Procedures

\begin{tabular}{|c|c|}
\hline Flat Sky Technique & Least Squares Solution \\
\hline assumes sky is flat $\left(S^{0}\right)$ & solves for real sky $\left(S^{\alpha}\right)$; will find $S^{0}$ if warranted \\
\hline requires dark frames & no dark frames needed, but they are useful if available \\
\hline $\begin{array}{l}\text { may take time for chopping to nearby } \\
\text { flat field region (if such exists) }\end{array}$ & no chopping needed \\
\hline confused fields can ruin the solution & confused fields can improve the solution \\
\hline $\begin{array}{l}\text { may remove flat emission components of the astronomical sky } \\
\text { (e.g. zodiacal emission, nebular emission, cosmic backgrounds) }\end{array}$ & preserves full sky intensity \\
\hline $\begin{array}{l}\text { requires Monte Carlo or ad hoc assessment of uncertainties } \\
\text { or unbiased source removal }\end{array}$ & $\begin{array}{l}\text { can accurately and analytically track } \\
\text { uncertainties and correlations }\end{array}$ \\
\hline $\begin{array}{l}\text { observations of } S^{0} \text { by all pixels automatically tightly } \\
\text { correlate all detector pixels on all spatial scales }\end{array}$ & $\begin{array}{l}\text { dithering must establish tight correlations between } \\
\text { all detector pixels }\end{array}$ \\
\hline computationally simple & can be simplified to produce the flat sky result \\
\hline & can be used in non-astronomical applications \\
\hline
\end{tabular}


Table 2. Figures of Merit for Single Pointings

\begin{tabular}{|c|c|c|c|c|c|c|c|c|c|c|}
\hline \multirow[b]{2}{*}{$M_{\text {pattern }}$} & \multicolumn{8}{|c|}{$256 \times 256$ arrays } & \multicolumn{2}{|c|}{$32 \times 32$ arrays } \\
\hline & Reuleaux & Random & VLA & Geometric & DASI & WIRE & NICMOS HDF-S & $2 \mathrm{MASS}$ & Reuleaux & Grid \\
\hline 6 & 0.002 & $\cdots$ & $0.020^{\mathrm{e}}$ & 0.016 & .. & .. & $\cdots$ & 0.002 & $\ldots$ & $\ldots$ \\
\hline 8 & & .. & & 0.058 & .. & . & . & & & $\cdots$ \\
\hline 9 & $0.003^{\mathrm{a}}$ & . & $0.059^{\mathrm{f}}$ & & . & .. & . & $\ldots$ & $0.217^{\mathrm{k}}$ & . \\
\hline 10 & $\cdots$ & $\cdots$ & $\ldots$ & 0.094 & $\cdots$ & $\cdots$ & . & $\cdots$ & $\cdots$ & $\cdots$ \\
\hline 12 & & & & 0.112 & & & & & & \\
\hline 13 & & $\cdots$ & & & 0.100 & & & & & $\cdots$ \\
\hline 14 & $\cdots$ & $\cdots$ & $\ldots$ & 0.139 & & $\cdots$ & $\cdots$ & $\cdots$ & $\cdots$ & $\cdots$ \\
\hline 15 & $0.117^{\mathrm{b}}$ & & $0.128^{g}$ & & $\cdots$ & $\cdots$ & .. & $\cdots$ & $\cdots$ & $\cdots$ \\
\hline 16 & & 0.162 & & 0.152 & .. & .. & .. & $\ldots$ & $\ldots$ & $\ldots$ \\
\hline 18 & $0.153^{\mathrm{c}}$ & 0.181 & 0.166 & 0.133 & $\ldots$ & $\ldots$ & . & $\ldots$ & $\ldots$ & $\ldots$ \\
\hline 24 & & & & 0.168 & & & & & & \\
\hline 27 & 0.265 & & $0.240^{\mathrm{h}}$ & & $\cdots$ & .. & $\cdots$ & & $\cdots$ & $\cdots$ \\
\hline 32 & & 0.286 & & 0.193 & & & & $\cdots$ & & $\cdots$ \\
\hline 39 & 0.307 & & 0.282 & & $\cdots$ & $\cdots$ & $\cdots$ & $\cdots$ & $\cdots$ & $\cdots$ \\
\hline 40 & & & & 0.193 & $\cdots$ & $0.228^{\mathrm{i}}$ & . & $\cdots$ & $\cdots$ & $\cdots$ \\
\hline 60 & 0.323 & 0.318 & 0.303 & 0.204 & $\cdots$ & $\cdots$ & $\cdots$ & & $\cdots$ & $\cdots$ \\
\hline 90 & 0.341 & 0.335 & 0.316 & 0.208 & & $\cdots$ & & $\cdots$ & & $\cdots$ \\
\hline 120 & 0.361 & 0.351 & 0.329 & 0.208 & & $0.225^{\mathrm{j}}$ & & & & \\
\hline 142 & & & & & & $\cdots$ & 0.131 & & & \\
\hline 150 & 0.387 & 0.365 & 0.347 & $\cdots$ & $\cdots$ & . & 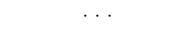 & $\cdots$ & $\cdots$ & $\cdots$ \\
\hline 180 & 0.416 & 0.378 & 0.366 & $\cdots$ & $\cdots$ & .. & $\cdots$ & & $\cdots$ & . \\
\hline 210 & 0.448 & 0.415 & 0.392 & $\ldots$ & $\cdots$ & . & $\cdots$ & $\ldots$ & $\ldots$ & . \\
\hline 240 & $0.485^{\mathrm{d}}$ & 0.437 & 0.419 & 0.212 & $\ldots$ & $\ldots$ & $\ldots$ & $\ldots$ & $\ldots$ & $\cdots$ \\
\hline 300 & 0.526 & 0.439 & 0.469 & & & & & & & \\
\hline 1024 & & & & & & & & & & 0.783 \\
\hline 4096 & $\cdots$ & $\cdots$ & $\cdots$ & $\cdots$ & $\ldots$ & $\ldots$ & $\cdots$ & $\cdots$ & $\cdots$ & 0.889 \\
\hline
\end{tabular}

Note. - Standard pattern sizes are: Reuleaux width $=128$ pixel, Random $3 \sigma=128$ pixel, VLA $r_{\max }=125.7$ pixel, Geometric is scaled to the 256 pixel array size, DASI $r_{\max }=82.5$ pixel, WIRE medium and deep surveys scaled by a factor of 2 , NICMOS HDF-S all camera 3 F110W data, $32 \times 32$ Reuleaux width $=12$ pixel, Grid spacing $=1$ pixel

$\mathrm{a}_{0} 0.037$ if $3 \%$ random variations added

$\mathrm{b}_{0.146}$ if $3 \%$ random variations added

${ }^{\mathrm{c}} 0.171$ if $3 \%$ random variations added

$\mathrm{d}_{0.396 \text { for } \text { width }}=110$ pixel, 0.513 for width $=144$ pixel

$\mathrm{e}_{r_{\max }}=16$ pixel

${ }^{\mathrm{f}} r_{\max }=32$ pixel

$\mathrm{g}_{r_{\max }}=125$ pixel

$\mathrm{h}_{0.176}$ for $r_{\max }=16$ pixel, 0.199 for $r_{\max }=16$ pixel

$\mathrm{i}_{0.065}$ for the unscaled pattern

$\mathrm{j}_{0.062}$ for the unscaled pattern

$\mathrm{k}_{0.162}$ for width $=8$ pixel

Table 3. Figures of Merit for Deep Surveys

\begin{tabular}{|c|c|c|c|c|c|c|c|c|c|c|c|c|}
\hline \multirow{2}{*}{$\begin{array}{l}\text { Survey } \\
\text { Size }\end{array}$} & \multirow{2}{*}{$\begin{array}{l}\text { Spacing } \\
\text { (pixels) }\end{array}$} & \multicolumn{2}{|c|}{ Reuleaux } & \multicolumn{3}{|c|}{ Random } & \multicolumn{3}{|c|}{ VLA } & \multicolumn{3}{|c|}{ Geometric } \\
\hline & & $M=15$ & 39 & $M=6$ & 16 & 40 & $M=6$ & 15 & 39 & $M=6$ & 16 & 40 \\
\hline $2 \times 2$ & 181 & 0.194 & 0.318 & 0.028 & 0.209 & 0.318 & 0.028 & 0.156 & 0.310 & 0.027 & 0.183 & 0.221 \\
\hline $2 \times 2$ & 218 & 0.173 & 0.314 & 0.016 & 0.191 & 0.313 & 0.022 & 0.168 & 0.303 & 0.022 & 0.174 & 0.216 \\
\hline $2 \times 2$ & 256 & 0.166 & 0.311 & 0.012 & 0.172 & 0.309 & 0.020 & 0.153 & 0.298 & 0.020 & 0.165 & 0.211 \\
\hline $3 \times 3$ & 181 & 0.198 & $\ldots$ & 0.020 & 0.213 & $\ldots$ & 0.028 & 0.160 & $\ldots$ & 0.027 & 0.187 & $\ldots$ \\
\hline $3 \times 3$ & 218 & 0.182 & $\ldots$ & 0.017 & 0.194 & $\ldots$ & 0.022 & 0.172 & $\ldots$ & 0.022 & 0.177 & $\ldots$ \\
\hline $3 \times 3$ & 256 & 0.170 & $\ldots$ & 0.016 & 0.178 & $\ldots$ & 0.020 & 0.158 & $\ldots$ & 0.020 & 0.168 & $\ldots$ \\
\hline $4 \times 4$ & 181 & $\ldots$ & $\ldots$ & 0.022 & $\ldots$ & $\ldots$ & 0.028 & $\ldots$ & $\ldots$ & 0.027 & $\ldots$ & $\ldots$ \\
\hline
\end{tabular}


Table 4. Figures of Merit for $4 \times 4$ Shallow Surveys

\begin{tabular}{|c|c|c|c|c|c|c|c|c|c|c|}
\hline \multirow{2}{*}{$\begin{array}{l}\text { Survey } \\
\text { Depth }\end{array}$} & \multicolumn{2}{|c|}{ Reuleaux } & \multicolumn{2}{|c|}{ Random } & \multicolumn{2}{|c|}{ VLA } & \multicolumn{2}{|c|}{ Geometric } & \multicolumn{2}{|c|}{ Grid } \\
\hline & $M=16$ & 32 & $M=16$ & 32 & $M=16$ & 32 & $M=16$ & 32 & $M=2$ & 3 \\
\hline 2 & 0.088 & 0.117 & 0.086 & 0.120 & 0.090 & 0.120 & 0.079 & 0.077 & 0.001 & \\
\hline 3 & 0.113 & 0.175 & 0.115 & 0.177 & 0.109 & 0.168 & 0.110 & 0.135 & $\ldots$ & 0.002 \\
\hline $3^{\mathrm{a}}$ & 0.182 & $\ldots$ & 0.165 & $\ldots$ & 0.167 & $\ldots$ & 0.136 & $\ldots$ & $\ldots$ & $\ldots$ \\
\hline
\end{tabular}

Note. - All surveys used 181 pixel grid spacing.

${ }^{a}$ Random rather than sequential selections from the dither patterns. 